\title{
Statistical Studies of the Robustness of the LFC Main Dipole Mechanical Structure
}

\author{
M. Bajko, P. Fessia, D. Perini \\ CERN, Geneva, Switzerland
}

\begin{abstract}
This paper describes two methods used to study the effect of the tolerances of the components on the structure of the LHC main dipole. The first mathod, called semi-statistical, is useful for the study of the effect of single different parameters and allows the determination of the acceptable variance of the dimensions of magnet components. The second one, fully statistical, allows the study of the combined effect of many parameters. The use of these two methods allowed to evaluate with good confidence the robustness of two different dipole cross-section designs, featuring austenitic and aluminium alloy collars, respectively.
\end{abstract}

\section{INTRODUCTION}

The Large Hadron Collider [1] requires 1232 twin-aperture superconducting (SC) main dipole magnets with a magnetic length at $1.9 \mathrm{~K}$ of $14.3 \mathrm{~m}$ and a nominal field of $8.3 \mathrm{~T}$.

The mecharical structure of the main dipole must, up to above the ultimate field of $9 T$ and in spite of electromagnetic forces reaching $400 \mathrm{t} / \mathrm{m}$, prevent any systematic coil movement causing quenches. A predictable and controllable structure is requited also to achieve the requited tolerances on field quality of a few $10^{-4}$. Throughout assembly at room temperature, cool down to $1.9 \mathrm{~K}$ and operation at high field, the different components exchange at their boundaries widely varying forces, which induce deformations of several tenths of a millimetre. In spite of this, the magnet must behave as a continuous, stiff structure. The design and assembly of components must in addition allow for tolcrances achievable in a cost-effective way, to be suitable for magnet series production at an affordable price.

It is therefore essential to verify that the chosen design is robust with respect to practical tolerances of components and their assembly. The behaviour of two possible dipole crosssections, design $A$ with aluminium alloy (AA) collars, and design $B$ with austenitic steel (AS) collars, were compared in detail by means of statistical methods which, together with the obtained results, are reported in this paper.

These designs are presented in some more detail in [2].

\section{Prohlem DefNITION}

Each industrially produced item reflects, in the difference between its actual dimensions and the theoretical ones, the applied production process. If the process is under statistical control and if there is no systematic bias because of tooling imperfections, the statistical distribution of the characteristics of the items can be foreseen on the basis of the known behaviour of the process itself. Under these conditions, the distribution is a purely normal distribution Manuscript neceived on 27 September 1999 with the mean centred on the theoretical value. This is the hypothesis made in the present work. Like the components, atso the assembly procedures are affected by their own indeterminacy. This can be estimated thanks to the statistical distribution of the observed assembly parameter values. The consequences of the tolerances of the components and of the assembly procedures of a structure determine the robustness and reliability of its design.

To estimate the above propertics, it is necessary to identify a set of design variables; for each of them an objective value, within an acceptability window, can be determined so as to achieve the required global performance.

It is therefore necessary to provide a tool to estimate the effect of component and assembly tolerance distributions on the design variable distributions. The tool must be abie to evaluate the reaction of the structure to the variation of one or a set of parameters at a time, and to statistically analyse the resulting perturbations.

Such a tool is a finite element (FE) model [3] of the structure. The software describing this model must be able to modify dimensions by reading data from an input file or an internal array and must rapidly compute the structure response. A minimum number of significant design variables is selected to keep the computing time within acceptable timits. The studicd dipole cross-section and the design variables are shown in Fig. 1 and Table $I$, respectively. To study the behaviour of the structure, the main tolerances along the horizontal and vertical axis of the magnet were considered. To sum tolerances, it was assumed that the statistical distribution of any dimension considered was independent from that of any other and that they are gaussian.

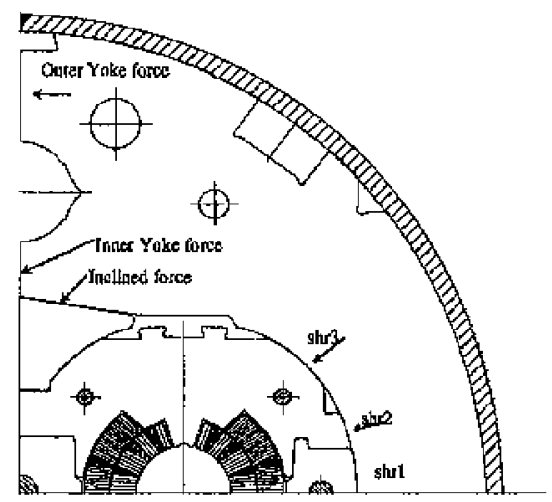

Fig. 1, LHC main dipole cross section showing the design variables. 
TABLEI

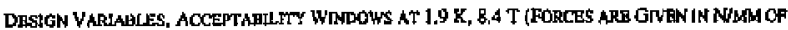
MALANT LENGTH) AND AFPECTRNG PARAMETRRS (MNOST IMPORTANT ONES IN BOLD)

\begin{tabular}{|c|c|c|}
\hline $\begin{array}{l}\text { Design } \\
\text { Variables } \\
\end{array}$ & $\begin{array}{l}\text { Acceptability } \\
\text { window }\end{array}$ & $\begin{array}{l}\text { Parameters affecting the design } \\
\text { variables }\end{array}$ \\
\hline $\begin{array}{l}\text { Outer } \\
\text { Yoke } \\
\text { Force }\end{array}$ & $>20 \mathrm{~N} / \mathrm{mm}$ & $\begin{array}{l}\text { Yoke, collar width, collar height, } \\
\text { cylinder stress, insert dimensions, } \\
\text { assembly tolerances }\end{array}$ \\
\hline $\begin{array}{l}\text { Inner } \\
\text { Yoke }\end{array}$ & $>20 \mathrm{~N} / \mathrm{mm}$ & $\begin{array}{l}\text { Yoke, collar width, collar height } \\
\text { cylinder stress, assembly }\end{array}$ \\
\hline Force & & tolerances \\
\hline $\begin{array}{l}\text { Inclined } \\
\text { force }\end{array}$ & $>20 \mathrm{~N} / \mathrm{mm}$ & $\begin{array}{l}\text { Yoke, collar width, collar height, } \\
\text { cylinder stress, assembly } \\
\text { tolerances }\end{array}$ \\
\hline $\begin{array}{l}\text { Shoulder } \\
(1,2,3)\end{array}$ & $>20 \mathrm{~N} / \mathrm{mm}$ & $\begin{array}{l}\text { Yoke, collar width, collar height, } \\
\text { cylinder stress, assembly } \\
\text { tolerances }\end{array}$ \\
\hline $\begin{array}{l}\text { Coil } \\
\text { azimuth. } \\
\text { pre-stress }\end{array}$ & $\begin{array}{l}>10 \mathrm{MPa} \\
<70 \mathrm{MPa}\end{array}$ & $\begin{array}{l}\text { Yoke, collar width, collar height, } \\
\text { cylinder stress, pole shims, } \\
\text { assembly tolerances }\end{array}$ \\
\hline
\end{tabular}

This allows to sum quadratically the distribution amplitudes at $1 \sigma$ as follows:

$$
\sigma=\sqrt{\sum_{i}^{n} \sigma_{i}^{2}}
$$

\section{IIL. SEMI-STATISTICAL APPROACH}

First it has been necessary to study the behaviour of the structure when changing the values of more than one parameter at the same time. The FE model was modified so as to compute in one run the design variables for several different dimensions of the components. The option of acting only on the contact elements was taken because it does not require re-meshing the whole model from one computation to the other, thus shortening the run time by about a factor two. A number of arrays equal to the number of parameters, the effects of which will be studied, is introduced. Each array describes the chosen interval of variation of each parameter in steps. To reduce errors, it is important to take the interval of variation slightly larger than the zone of interest.

The problem is then solved for all the possible combinations of parameters. For $m$ parameters, each one taking $n$ different values, the problem is made up of $n^{\mathrm{m}}$ cases, for which the structure response has to be computed. The results, for each of the design variables, are then postprocessed by a Mathematica ${ }^{\text {TM }} 1$ program.

The results concerning each design variables are first interpolated to generate a transfer function. This function represents a good approximation of the behaviour of the structure when varying the selected $m$ parameters.

To evaluate the robustness and stability of the structure, $m$ probability distribution curves are then introduced,

\footnotetext{
${ }^{1}$ Mathematica is a trademark of Wolfram Research Inc.
}

representing the tolerance distribution around the nominal values. Each parameter can be represented by different probability distributions resulting from different production processes, but in the computations reported in this article, all the distributions were assumed to be gaussian. Obviously each distribution will be characterised by a different

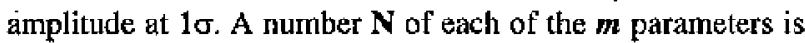
then extracted according to the $\boldsymbol{m}$ distributions, and injeeted in the transfer functions to get the corresponding value of the design variables. The $\mathbf{N}$ obtained values are then analysed to identify which percentage of them falls inside the interval of acceptability. Fig. 2 and 3 . show the probability for the strength of the mating force along the inner gap between the two yoke halves, when the inner and outer gap dimensions are varied at the same time. The computations show clearly how design $A$ is much more sensitive to effect of tolerances than design $\mathrm{B}$ (tolerances amplitude distribution at $1 \sigma=0.04$ $\mathrm{mm}$ and $\mathrm{N}=4000$ ).

Moreover, the method can also be used to determine which is the tolerance interval of (a) given parameter(s) for which the design variable stays inside the defined window of acceptability. The computations performed to get the

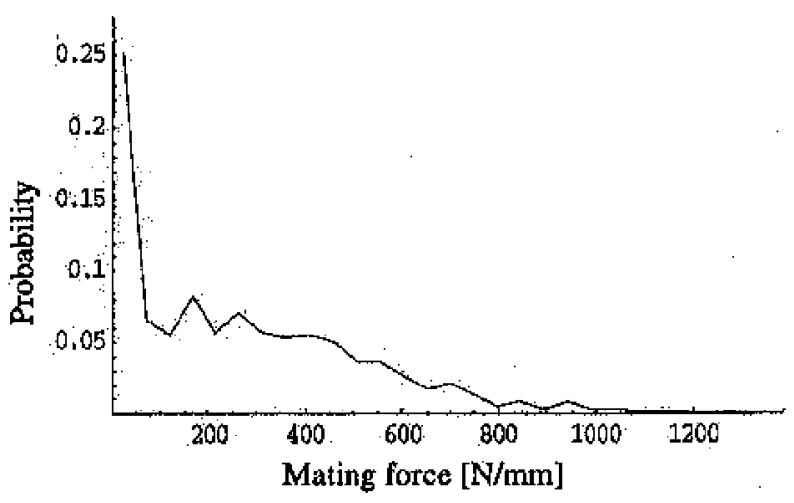

Fig. 2. Design A, AA collars. Probability distribution of the value of the mating force along the inter yoke gap, for variations of the innes and outer gap dimensions ( $1 \sigma=0.04 \mathrm{~mm}$ )

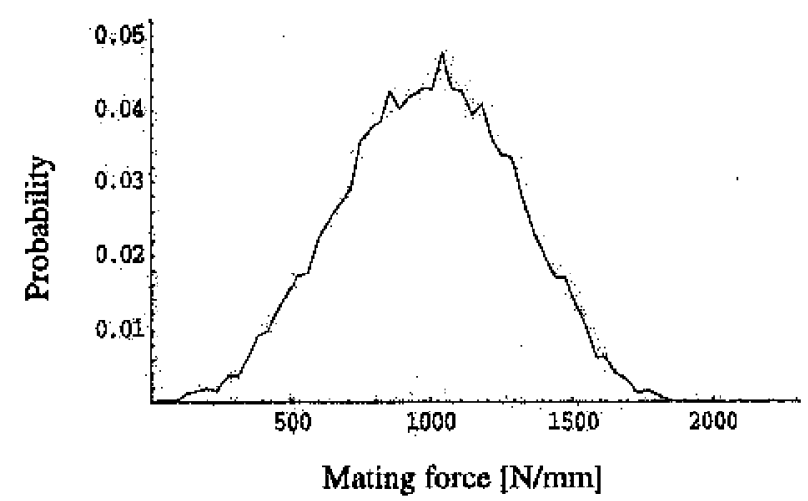

Fig. 3. Design B, AS collars. Probability distribution of the value of the mating force along the inner yoke gap, for variations of the inner and outer gap dimensions $(1 \sigma=0,04 \mathrm{mr})$ 


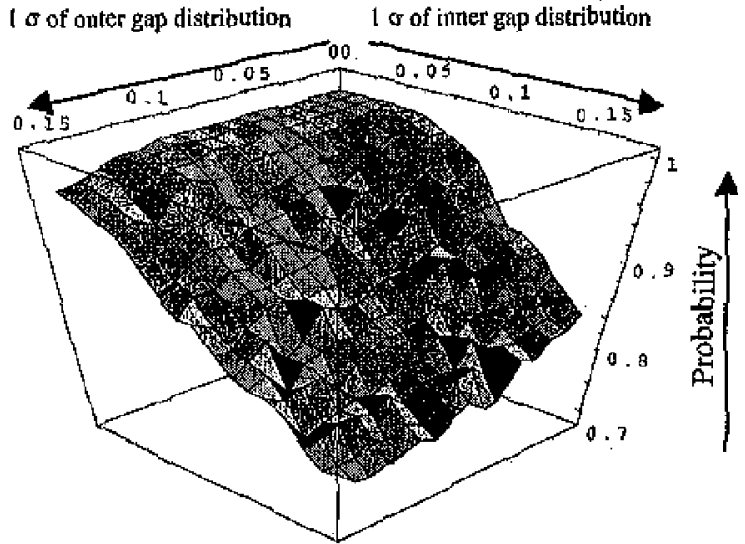

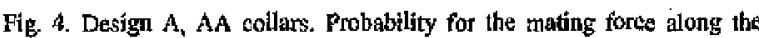
juner yoke gap to fall inside the aceeptability window (>20N/mm) as function of the dispersion $\sigma$ of the toleranees of the inner and outer gaps.

probability distributions of the forces are repeated varying the amplitude of the parameter distribution at $1 \sigma$. For each computed probability distribution, the cumulative probability that the design parameter falls inside the window of acceptability can then be estimated. By plotting this result against the corresponding width at $1 \sigma$, it is possible to obtain a good estimation of the maximum admissible tolerance width.

Fig. 4 and 5 show the probability that the force exchanged along the inner gap of the magnet is larger than zero for design $A$ and design $B$, respectively. The latter presents a considerably better robustness: the maximum acceptable uncertainty in the dimension of the yoke inner and outer gap (resulting from the quadratic addition of the tolerances of the collars, yoke laminations and their assembly procedures) is of the order of $0.1 \mathrm{~mm}$ while for design A this uncertainty is only $0.04 \mathrm{~min}$.

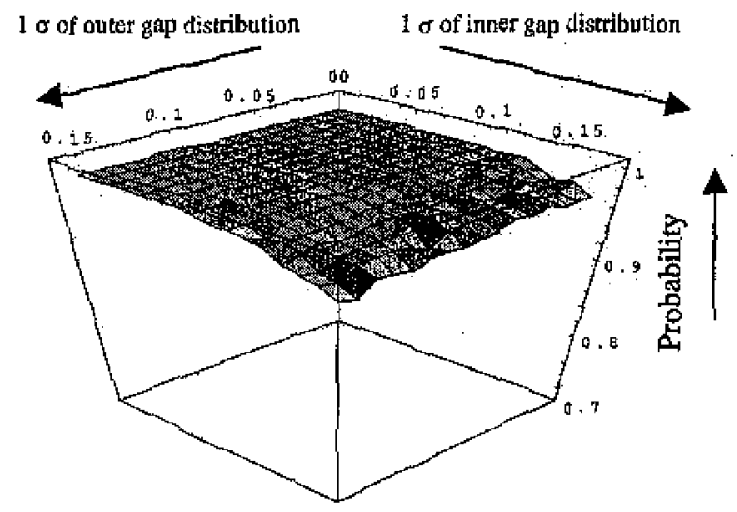

Fig. 5, Design B, AS collars. Probability for the mating force along the inner yoke gap to fall inside tho window of acceptabiticy ( $202 \mathrm{~N} / \mathrm{mm}$ ) as function of the dispersion or of the tolerances of the ijuner and outer yoke gaps

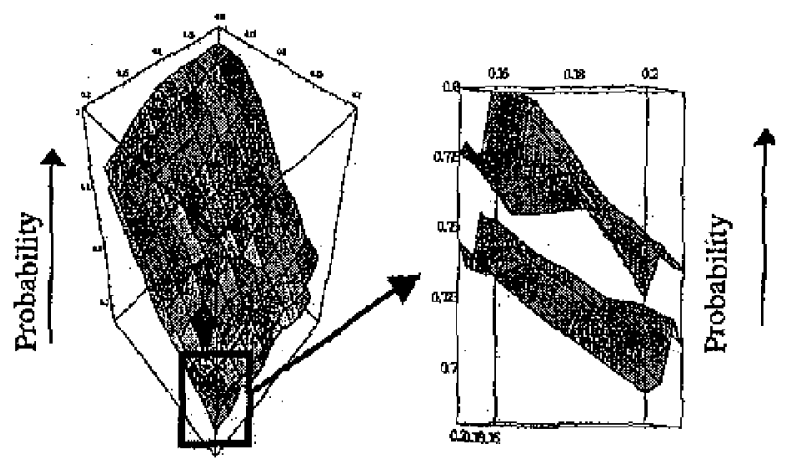

Fig. 6. Design A, AA collars. Probability for the mating force along the itmer yoke gap to fall inside the acceptability window (>20N/mm) as funtion of the dispersion of the tolerances of inner and onter gap. Upper surface ind. Yoke $\sigma=0.02 \mathrm{~mm}$ and cylinder stress $\sigma=5 \mathrm{MPa}$. Lower surface ind, Yoke $\sigma=0.1 \mathrm{~mm}$ and cylindor stress $\sigma=25 \mathrm{Mpa}$.

A family of curves can be represented as in Fig. 6, where the toletances affecting the shrinking cylinder stress (induced by welding) and the tolerances of the insert between yoke and collars were introduced.

Comparing Fig. 4 and Fig. 6, it can be seen that, if more parameters are added, then the range where the force has acceptable values is even smaller because of the uncertainties of the additional parameters.

The method described in the previous chapter is advantageous when the aim is to understand the behaviour of up to 4 or 5 parameters at the same time and when it is important to understand what is the effect of an increase of the amplitude of the tolerance distribution. On the other hand, for larger number of parameters and if the aim is to have a finer estimation of the structure robustness, a full statistical approach has to be used. In this case $\mathbf{N}$ computations are performed in the FE model, each one with a different group of $\mathbf{m}$ values extracted according to their defined statistical distribution. For the work presented here, the input values are brought into the $\mathrm{F} E$ model from an ASCII file originated by an appropriate Mathematica ${ }^{\mathrm{TM}}$ code (their extraction from the ANSYS FEM model was rejected because there the seet of the random number generator is hard coded and therefore the extracted sequence was always the same). The FE results are then saved in an ASCII file. This file is read by a Mathematica ${ }^{\text {TM }}$ program to study the statistics of the results. The adopted method is a pure Monte Carlo analysis. No methods to reduce the variance (survival biasing, exponential transformation) were used.

The results obtained (Fig. 7) confirm the indication resulting from the semi-statistical approach. The much larger robustness of design B (AS collars) with regard to that of design A (AA collars) is absolutely clear. For the latter, the probability to have an open inner yoke gap at nominal field (and hence an unstable behaviour) concerns $25 \%$ of the production (this figure is less than $0.1 \%$ for design B). In these computations, the tolerance distributions around the 
nominal values were the same for both designs, and each of them had been optimised before 80 as to have the best possible behaviour. This is a conservative approach for design $B$, where the collared coil dimensions are less dependent on coils pre-stress than for the design $A$, for which in addition the yoke gap has to be controlled to within $\pm 0.05 \mathrm{~mm}$ over a $15 \mathrm{~m}$ length when welding the magnet shrinking cylinder. This latter control is not required for design $\mathrm{B}$, since the yoke gap is closed during welding.

Steel

Outer Yoke contact [ $[\mathrm{Y} / \mathrm{mm}]$
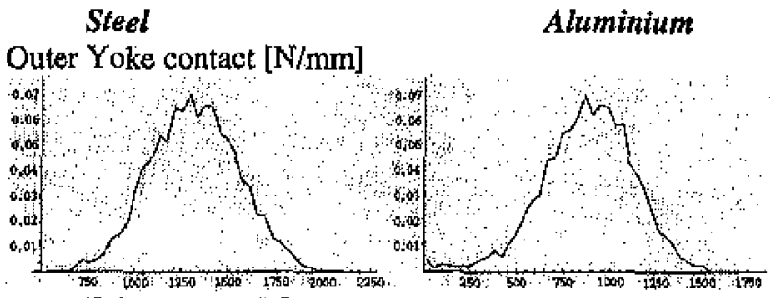

Inner Yoke contact [N/mm]

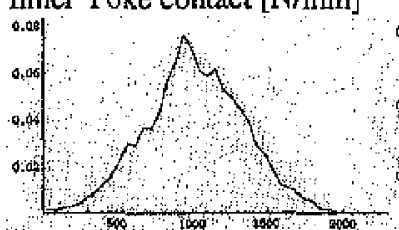

Inclined Yoke contact [N/mm]

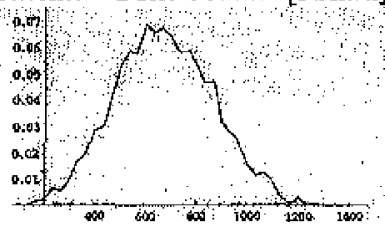

Cylinder stress [MPa]

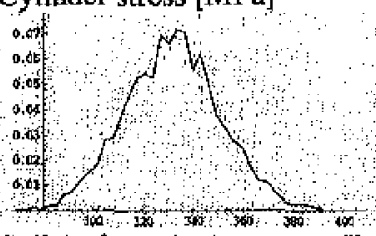

Coil (pole region) stress [MPa]
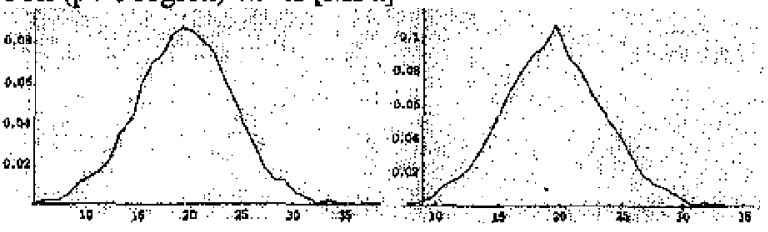

Fig.7. Resuits of the Monte Carlo study. Comparison of the probability distributions of main design variables for design B (AS collars) and design $\mathrm{A}$ (AA coll $\mathrm{ars}$ ) at $1.8 \mathrm{~K}$ and $8.4 \mathrm{~T}$.

\section{ERROR ESTIMATION FOR THE MONTE CARLO METHOD}

An estimation of the error of the computations reported here can be made by determining the Percentage Relative Standard Deviation. (P.R.S.D.). This gives an indication of the reliability of the estimation of the mean of the distribution. According to the theorem of the central value, the P.R.S.D. should follow the $\mathrm{N}^{1 / 2}$ law. In Fig. 8 the P.R.S.D. of the median value of the force exchanged along the outer yoke interface is plotted together with the $\mathrm{N}^{-1 / 2}$ line showing good agreement. The fnal value is $1.7 \%$. This means that the mean value of this force, estimated to be 891 $\mathrm{N} / \mathrm{mm}$, will be within an interval of $\pm 31 \mathrm{~N} / \mathrm{mm}$ with a probability of 0.9544 .

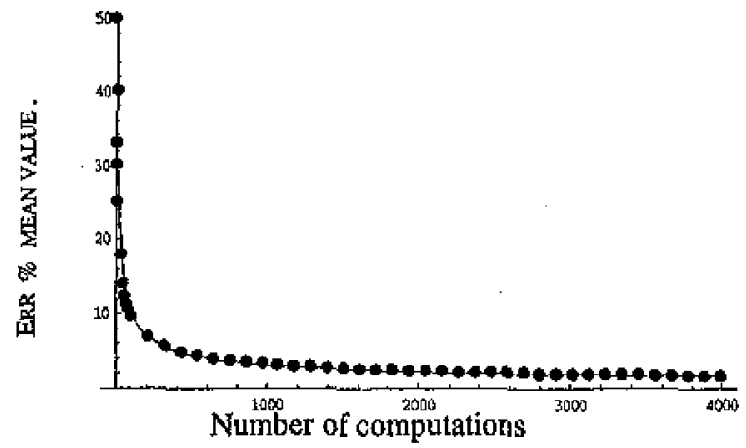

Fig. 8. Theoretical and computed P.R.S.D. of the median value of the mating force at outer yoke interface versus the number $N$ of compited cases

\section{CONCLUSION}

Two statistical methods to study the robustness and stability of the mechanical structures of the L.HC main dipoles have been implemented. Both of them give coherent indications and provide complementary insight on the same problem. The computations performed comparing a structure with austenitic collars and closed yoke gap and another with aluminium collars and open yoke gap showed clearly that the first one is much more robust than the second one. In addition to contributing to the selection of the dipole crosssection design for series manufacture, the developed statistical evaluation tools can be used to define the largest allowable tolerances for components, with a view to simplify their manufacturing process and thereby reduce costs.

\section{AKNOWLEDGMENT}

The authors thank C. Wyss for his support and E. Todesco for fruitful discussions.

\section{REFERENCES}

[1] The Large Hadron Collider Conceptual Design, CERN-AC 95-05

[2] P. Fessia et al, "Selection of the cross-section design for the LHC main dipole", this Conference

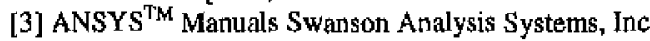

